\title{
Norois
}

Environnement, aménagement, société

$233 \mid 2014$

Mobilité, santé et développement territorial : de nouveaux défis pour la gouvernance des territoires ruraux

\section{Mobilités des jeunes adultes et politiques d'accueil dans les territoires ruraux. Etudes de cas en France et au Québec}

Young adults mobilities and policies for welcoming in rural areas. Cases studies

in France and Quebec

Émilie Jamet, Patrice LeBlanc et Sylvie Lardon

\section{OpenEdition}

Journals

Édition électronique

URL : https://journals.openedition.org/norois/5297

DOI : $10.4000 /$ norois. 5297

ISBN : 978-2-7535-4127-6

ISSN : $1760-8546$

Éditeur

Presses universitaires de Rennes

Édition imprimée

Date de publication : 20 décembre 2014

Pagination : $37-51$

ISBN : 978-2-7535-4083-5

ISSN : 0029-182X

Référence électronique

Émilie Jamet, Patrice LeBlanc et Sylvie Lardon, « Mobilités des jeunes adultes et politiques d'accueil dans les territoires ruraux. Etudes de cas en France et au Québec », Norois [En ligne], 233 | 2014, mis en ligne le 20 décembre 2016, consulté le 14 janvier 2022. URL : http://journals.openedition.org/ norois/5297 ; DOl : https://doi.org/10.4000/norois.5297 


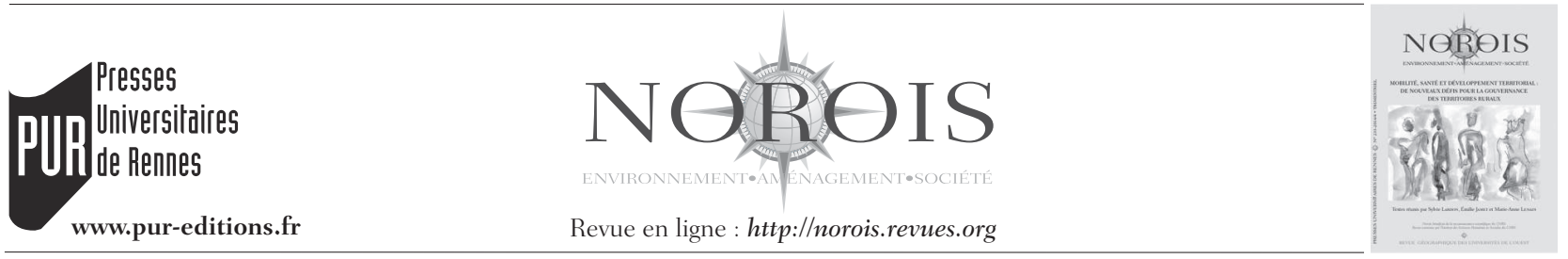

\title{
Mobilités des jeunes adultes et politiques d'accueil dans les territoires ruraux - Études de cas en France et au Québec
}

\author{
Young Adults Mobilities and Policies for Welcoming in Rural Areas \\ Cases Studies in France and Quebec
}

\author{
Émilie Jamet ${ }^{* a}$, Patrice LeBlanc ${ }^{b}$ et Sylvie LARDON ${ }^{a}$
}

\footnotetext{
*Auteur correspondant

a UMR Métafort, INRA/AgroParisTech, 9 avenue Blaise-Pascal, CS 20085 - 63178 Aubière, France (emjamet@

gmail.com) (sylvie.lardon@agroparistech.fr)

${ }^{b}$ Chaire Desjardins en développement des petites collectivités, Université du Québec en Abitibi-Témiscamingue,

445 boulevard de l'Université - Rouyn-Noranda (Québec), Canada - J9X 5E4 (Patrice.Leblanc@uqat.ca)
}

Résumé : Cet article a pour objectif d'alimenter les connaissances sur les migrations ville-campagne des jeunes adultes. Il éclaire la manière dont leurs parcours migratoires structurent leurs rapports aux mobilités comme aux territoires ruraux. Il se penche aussi sur les politiques d'accueil ou de mobilité mises en œuvre localement pour accompagner ces processus. Nous mobilisons les principes de la théorie ancrée pour analyser deux études de cas, dans le Pays de Saint-Flour Haute Auvergne (France) et au Témiscamingue (Québec). Nous étayons l'hypothèse selon laquelle les départs des jeunes adultes depuis les territoires ruraux ne sont pas nécessairement synonymes de rupture de l'attachement ou de manque d'implication des jeunes dans les territoires ruraux. Nous suggérons que les politiques d'accueil peuvent être associées à des initiatives, conduites dans les territoires ruraux, visant à développer chez les jeunes adultes, des apprentissages liés aux mobilités pour mieux contribuer aux dynamiques des territoires. Les concepts de « motilité » et de « capital spatial des territoires » apparaissent opérants à la fois pour renouveler la compréhension du phénomène de migration des jeunes depuis les territoires ruraux, mais également pour repenser les actions locales pour l'accompagnement des mobilités des jeunes ou leur accueil dans les territoires ruraux.

\begin{abstract}
This article aims to develop knowledge about rural-urban migration of young adults. We show how their migration routes structure their relationships to mobility as to rural areas. We also analyze how local welcoming and mobility policies support these processes. We mobilize the principles of grounded theory to analyze two case studies, in the Pays de Saint-Flour Haute Auvergne (France) and Témiscamingue (Québec). We support the hypothesis that the departure of young adults from rural areas does not necessarily mean breaking the attachment or a lack of involvement of young people in rural areas. We suggest that welcoming policies can be based on initiatives, conducted in rural areas, to develop learning concerning mobility and to better contribute to territorial dynamics. The concepts of "motility" and "spatial capital of territory» appear as a good way to renew the understanding of the phenomenon of migration of young people from rural areas, and also to rethink local actions to support young mobilities or welcoming policies.
\end{abstract}

Mots clés : parcours migratoires - récits de vie - faible densité de population - motilité - capital spatial des territoires - France, Québec

Keywords: migration routes - life stories - low population density - motility - spatial capital of territories - France - Quebec 


\section{INTRODUCTION : MOBILITÉS ET ACCUEIL DE JEUNES ADULTES DANS LES TERRITOIRES RURAUX}

Depuis les années 1970-1980, en France comme au Québec, des territoires ruraux de plus en plus éloignés des villes connaissent un regain démographique (Roussel et Mamdy, 2001 ; Cognard, 2010; Pistre 2011, 2012; Simard et Guimond, 2010; Guimond, 2012). Dans ceux qui composent avec de faibles densités de population ${ }^{1}$ (Gambino, 2008; Barthe et Milian, 2011) et sont éloignés des villes, la croissance démographique se pose comme un enjeu prioritaire. Après des décennies d'exode rural, «l'arrivée de nouvelles populations est considérée, par [les] territoires [touchés par l'exode rural], comme un phénomène positif » (Guérin, 2001), susceptible de contribuer aux dynamiques de développement territorial, et ainsi permettre « d'assurer la satisfaction des besoins et l'épanouissement des populations, tout en poursuivant des objectifs d'équité sociale, de ménagement de la nature, d'innovation économique et sociale et de participation démocratique » (Jean, 2006).

Dans le but d'accroître ou de maintenir le nombre d'habitants, des politiques territoriales d'accueil et de maintien de la population sont mises en œuvre. Elles visent à ralentir le vieillissement de la population, à pourvoir des besoins en main-d'œuvre, à maintenir des écoles ouvertes. Les jeunes adultes comptent parmi les profils particulièrement recherchés. Or, ces derniers sont souvent amenés à quitter les territoires ruraux pour des durées variables afin de poursuivre des études, d'occuper un emploi, de rejoindre un-e conjoint-e... Ces départs ne sont pas sans poser question aux élus et acteurs locaux qui misent sur une augmentation et un rajeunissement de la population pour assurer le développement de leurs territoires.

David (2014) souligne que «les connaissances portant spécifiquement sur les jeunes vivant en milieu rural n'ont pas vraiment été renouvelées depuis le début des années 1990 ». Cet article a

1. Nous n'avons pas caractérisé la faible densité au recours d'un critère statistique, qui aurait d'ailleurs été très différent en France et au Québec. Les territoires que nous avons étudiés comptent toutefois parmi les moins denses de leur contexte national (France) ou provincial (Québec).

Ils se caractérisent par un point commun mis en évidence par Barthe et Milian, 2011, p. 141 : « [Des] espaces marqués par la rareté des hommes et souvent aussi par celle des ressources financières pour les collectivités concernées. » pour objectif d'y contribuer, sans toutefois s'appuyer sur un clivage entre l'urbain et le rural. Il éclaire la manière dont les parcours migratoires des jeunes adultes structurent leurs rapports aux mobilités comme aux territoires ruraux. Il analyse aussi les politiques d'accueil ou de mobilité mises en œuvre localement pour accompagner ces processus. Nous étayons l'hypothèse selon laquelle les départs ne sont pas nécessairement synonymes de rupture de l'attachement ou de manque d'implication des jeunes dans les territoires ruraux. Réciproquement, des parcours sédentaires peuvent aussi être source d'isolement et de non-participation des jeunes aux dynamiques sociales, économiques, culturelles... Nous suggérons alors que les politiques d'accueil puissent être associées à des initiatives, conduites dans les territoires ruraux, visant à développer chez les jeunes adultes, des apprentissages liés aux mobilités pour mieux contribuer aux dynamiques des territoires.

Après une description des deux terrains d'étude et des méthodes employées, nous présentons quatre types de parcours migratoires des jeunes adultes observés. Ils combinent différents rapports aux territoires, ruraux et urbains, et à la mobilité.

Nous détaillons ensuite les dispositifs en faveur de l'accueil, du maintien et de la mobilité des jeunes adultes mis en œuvre dans chacun de nos territoires d'étude. Nous les distinguons selon qu'ils permettent de favoriser les arrivées et les retours et de pérenniser les installations, de laisser partir mais aussi permettre de revenir, d'accompagner les départs ou de favoriser les apprentissages par la mobilité.

À partir de ces résultats, nous discutons alors la manière dont la mobilité peut être une ressource tant pour les jeunes adultes que pour les territoires ruraux. Pour cela, nous mobilisons les notions de motilité (Kaufman, 2005) et de capital spatial territorial (Lévy, 2013) pour rendre compte de nos observations et proposer un renouvellement du regard des politiques territoriales sur la mobilité des jeunes adultes. 


\section{UNE MISE EN PERSPECTIVE DE DEUX Territoires : Pays de Saint-Flour Haute Auvergne (France) et TÉmiscamingue (QuÉBEC)}

Les résultats présentés et discutés dans cet article sont issus d'un travail de doctorat sur le rôle des parcours migratoires des jeunes adultes dans le développement des territoires ruraux ${ }^{2}$. Deux territoires ruraux de faible densité de population sont étudiés : le Pays de Saint-Flour Haute Auvergne, en France et le Témiscamingue, au Québec. Le choix de ces deux terrains ne s'inscrit pas dans une démarche comparative au sens classique, où il s'agit de « relever des différences et des points communs en fonction d'un critère défini au préalable » (Vigour, 2005) afin de vérifier des hypothèses préalablement définies. C'est une autre manière de comparer que nous avons retenue, dans la mesure où « l'objet à comparer n'est pas fixé mais à découvrir et porte autant sur les dissimilarités que sur les similarités »(Soulet, 2010).

À plusieurs reprises, des élus, agents de développement et acteurs socio-économiques du Pays de Saint-Flour Haute Auvergne et du Témiscamingue ont échangé au cours de voyages d'études ${ }^{3}$. Bien que situés dans des contextes politico-administratifs distincts, les acteurs, présents lors des échanges, ont constaté qu'ils faisaient face aux mêmes difficultés pour penser leurs projets de développement tout en prenant en compte le phénomène de migration des jeunes depuis leur territoire. C'est sur cet échange naissant entre deux territoires que nous avons empiriquement basé notre recherche. Nous avons cherché à mettre en perspective deux territoires partageant un même questionnement sur la mobilité des jeunes pour apporter des éléments de connaissance, mobilisables pour la mise en ouvre d'initiatives favorisant la participation des jeunes adultes dans les dynamiques territoriales, qu'elles soient sociales, économiques, culturelles ou environnementales.

2. Jamet E., Le rôle des parcours migratoires dans le développement des territoires ruraux. Le cas des jeunes adultes dans le Pays de Saint-Flour Haute Auvergne et au Témiscamingue. Travail de doctorat en cours débuté en 2010 sous la direction de Sylvie Lardon et Patrice LeBlanc.

3. À deux reprises, en 2009 et 2010 , et à la suite de collaborations entre des chercheurs de l'UQAT et d'AgroParisTech avec qui ils travaillaient, des agents de développement et des élus du Témiscamingue se sont rendus dans le Pays de Saint-Flour Haute Auvergne pour échanger sur leurs problématiques de développement.

\section{Pays de Saint-Flour Haute Auvergne et Témiscamingue : deux territoires ruraux éloignés des villes}

Le Pays de Saint-Flour Haute Auvergne et le Témiscamingue sont des territoires ruraux, peu denses et éloignés géographiquement des grandes agglomérations et de leur influence. Le Pays de SaintFlour Haute Auvergne (figure 1 - planche I) est un territoire de moyenne montagne situé dans le département du Cantal (Région Auvergne) qui figure parmi les six départements les moins peuplés de France (Insee, 2013a). La densité de population moyenne du territoire est de 16,3 habitants $/ \mathrm{km}^{2}$ en $2009^{4}$ (Insee, 2013b). Seules trois communes, Saint-Flour, Murat et Neussargues-Moissac, ont une densité de population supérieure à la moyenne nationale (Insee, 2009). En 2009, on comptait 37830 habitants dans le Pays dont 9729 dans la ville de Saint-Flour, sous-préfecture du département.

Le Témiscamingue (figure 2 - planche II) est une Municipalité Régionale de Comté (MRC) située dans la Région Abitibi-Témiscamingue, limitrophe de la Province de l'Ontario à l'Ouest du Québec. La MRC est composée de 21 municipalités dont la plupart appartient à des zones sans influence métropolitaine ou à influence faible ou modérée (Statistiques Canada, 2002). En 2013, le Témiscamingue compte 16346 habitants, soit $11 \%$ de la population d'Abitibi-Témiscamingue représentant elle-même 1,8\% de la population de la Province du Québec (Institut de la Statistique du Québec, 2014). La ville la plus importante de la MRC est Ville-Marie, qui comptait 2595 habitants en 2011.

En plus d'être faiblement peuplés, les deux territoires à l'étude ont la caractéristique commune de voir leur population décroître : -3 \% entre 1999 et 2009 pour le Pays de Saint-Flour Haute Auvergne et $-8,2 \%$ de 2001 à 2013 pour le Témiscamingue. Si un regain d'attractivité est observé dans le Pays de Saint-Flour Haute Auvergne comme dans le reste du Cantal, celui-ci reste insuffisant pour compenser la perte de population due à un solde naturel négatif (Insee, 2013b). Au Témiscamingue, en revanche, le solde migratoire reste globalement négatif sur la période 2001-2013, même si pour la première fois en 2013, la MRC a connu un solde

4. La densité de population moyenne en France métropolitaine est de 64,3 habitants $/ \mathrm{km}^{2}$ (Insee, 2013). 
migratoire quasi nul, ne perdant que 4 habitants (Observatoire de l'Abitibi-Témiscamingue, 2014a). Concernant la structure de la population, la part des moins de 30 ans est supérieure à celle des plus de 60 ans au Témiscamingue et cela malgré un départ des jeunes, notamment des 14-25 ans, plus marqué que pour les autres tranches d'âge (Observatoire de l'Abitibi-Témiscamingue, 2014b). Dans le Pays de Saint-Flour Haute Auvergne, le vieillissement de la population est fortement marqué : les plus de 60 ans représentent une part plus importante que celle des moins de 30 ans. Comme au Témiscamingue, ce sont également les jeunes, et notamment les 18-25 ans, qui sont la tranche d'âge la plus déficitaire dans le Pays de Saint-Flour Haute Auvergne, à l'image du reste du Cantal (Insee, 2013a).

Souhaitant relever le défi démographique, les acteurs de nos deux territoires d'étude, à l'instar d'autres régions rurales, en France comme au Québec, s'interrogent sur les éléments qui expliquent le départ des jeunes, mais également sur ceux qui peuvent favoriser leur arrivée ou leur retour. Le présent article a pour but d'alimenter ces interrogations " de terrain », mais aussi de contribuer à accroître les connaissances portant sur les jeunes vivant ou ayant vécu dans des territoires ruraux. Nous nous intéressons aux parcours migratoires des jeunes adultes - c'est-à-dire aux différents endroits qu'ils habitent au cours de leur vie et aux raisons qui expliquent les changements - et aux actions menées au sein des territoires qui peuvent freiner les départs, les accompagner ou favoriser l'accueil ou les retours. Notre travail s'inscrit dans le courant de recherche portant sur la migration des jeunes, notamment depuis et vers les territoires ruraux ${ }^{5}$ et celui sur les migrations villes-campagnes ${ }^{6}$.

5. C'est plus précisément dans l'optique des travaux conduits, au Québec, par le Groupe de Recherches sur la Migration des Jeunes (GRMJ) que nous inscrivons notre travail, et en France, par le travail de doctorat conduit par Gambino (2008) sur les modes d'habiter des jeunes dans les territoires de faible densité.

6. Notamment dans le courant des travaux présentés sur la thématique lors de la session spéciale « Migrations ville-campagne : dynamiques territoriales comparées » de l'ASRDLF 2011 , du colloque « Politiques d'accueil et mobilités dans les territoires ruraux. L'action publique face aux nouvelles géographies des modes de vie » (Lyon, 2011), ou des recherches conduites par le groupe de recherche québécois sur les migrations villecampagne et les néoruraux.

\section{Une posture empirico-déductive et entretiens compréhensifs}

Afin de répondre aux questions posées par les acteurs du terrain, nous avons opté pour une « posture d'enquête empirico-inductive ayant pour but la formulation de théories ancrées dans la production et l'analyse progressive de données de terrain » (Paillé, 2010). Nous revendiquons donc comme point de départ de la réflexion une « réalité locale et contextuelle » que nous avons tenté de « hisser à un niveau théorique par un travail méthodique de terrain » (Paillé, 2010). Nous n'avons pas cherché à vérifier une théorie formulée à partir d'une exploration de la littérature, mais à expliquer les phénomènes observés sur le terrain (Dumez, 2012 cité par Robineau, 2013).

Des séjours en immersion sur les territoires d'étude se sont avérés primordiaux pour atteindre un triple objectif (Gambino, 2008) : se créer une place au sein de nos terrains d'étude, décentrer notre regard pour ne pas focaliser sur nos a priori et être en interaction avec les acteurs du terrain pour vérifier nos interprétations. Au cours de ces séjours, des entretiens compréhensifs (Kaufman, 2007) menés de façon systématique ont été réalisés. Nous en distinguons trois types : les entretiens conduits avec des jeunes adultes, avec des acteurs intermédiaires et avec des élus.

\section{Entretiens conduits avec les jeunes adultes}

Nous avons cherché à comprendre les raisons pour lesquelles les jeunes adultes partent, viennent ou reviennent s'installer dans les deux territoires étudiés. Si la principale classe d'âge déficitaire dans les territoires étudiés s'étend jusqu'à 25 ans, il nous a semblé pertinent de ne pas nous arrêter à cette limite, de manière à prendre compte les parcours de vie des individus sur un pas de temps plus large que celui de la jeunesse absente de la campagne. Nous avons donc rencontré, lors d'entretiens compréhensifs, une trentaine de jeunes adultes âgés de 18 à 35 ans. Le récit de leur parcours migratoire a occupé une place centrale dans les échanges. En effet, nous leur avons demandé de nous raconter les différents lieux où ils ont habité depuis l'enfance et les raisons pour lesquelles ils ont déménagé. Les éléments de récit, c'est-à-dire « les moments où les enquêtés 
racontaient sous la forme narrative des épisodes de leur expérience vécue » (Bertaux, 2010) sont principalement ceux que nous avons mobilisés pour l'analyse. Les profils des jeunes adultes rencontrés en entretien étaient variés. Ils pouvaient ne jamais avoir quitté le territoire, en être parti puis revenu, habiter toujours à l'extérieur ou encore venir d'une autre région. Nous avons également été vigilants à rencontrer des personnes vivant dans différents lieux des territoires d'étude et aux profils sociodémographiques diversifiés : des jeunes sans ou avec diplômes (du secondaire jusqu'au doctorat), des personnes en couple avec ou sans enfants, d'autres célibataires, des jeunes occupant des emplois dans les secteurs primaire, secondaire ou tertiaire et des personnes sans emploi.

Chacun des entretiens a été enregistré, transcrit puis codé, d'abord de manière très descriptive, en utilisant des formulations proches du vocabulaire utilisé par les enquêtés. Au fur et à mesure des nouveaux entretiens, nous avons synthétisé un certain nombre de codifications à travers des intitulés plus génériques, regroupant de plus larges passages de verbatims, dégageant ainsi des profil-types de parcours migratoires. Au-delà des arguments justifiant le maintien ou les mobilités entre plusieurs territoires, nous nous sommes également attachés à saisir la dimension spatiale et temporelle des parcours migratoires.

\section{Entretiens conduits avec les acteurs intermédiaires et les élus}

Il était aussi indispensable de saisir les représentations des « autres » acteurs des territoires qui qualifient souvent le phénomène de migration des jeunes adultes comme une difficulté, voire une contrainte pour le développement. Une quarantaine d'entretiens compréhensifs a donc été conduit avec des élus et avec des acteurs intermédiaires, c'est-à-dire des personnes ayant à la fois une connaissance de l'action publique menée localement et un contact relativement direct avec les jeunes adultes du territoire. Dans ce cadre, nous avons rencontré des agents de développement, des chargés de mission (accueil, jeunesse, culture, installation, participation citoyenne), des travailleurs sociaux, des enseignants ou encore des conseillers en emploi.
Ces entretiens avaient deux objectifs principaux. Le premier visait à comprendre en quels termes, par rapport à quels questionnements les « acteurs du développement » (élus et acteurs intermédiaires) qualifient le phénomène de migration des jeunes. Le second objectif était de recenser les actions mises en œuvre dans les territoires pour freiner, accompagner, favoriser les départs ou les arrivées de jeunes adultes.

Comme pour les entretiens conduits avec les jeunes adultes, nous avons utilisé le même système de codification pour analyser les entretiens enregistrés et transcrits.

\section{JEUNES ADULTES : UNE DIVERSITÉ DE RAPPORT À LA MOBILITÉ ET AU TERRITOIRE}

Les quatre types de parcours migratoires présentés dans cette partie sont issus du travail de codification réalisé à partir des entretiens. Une telle synthèse est nécessairement réductrice de la singularité des cas de chaque individu. Il importe donc de garder à l'esprit que certains parcours individuels peuvent se situer à l'interface de ces différentes catégories que nous proposons. Ces résultats corroborent, sans toutefois complètement se confondre aux trois modalités de mobilités géographiques des jeunes - mobilité locale, mobilité alternante, mobilité pour la sédentarité mises en avant par Gambino (2008, 2010).

\section{Des parcours migratoires sédentaires}

Dans le premier type de parcours, les jeunes adultes rencontrés expliquent n'avoir pas ou peu déménagé au cours de leur vie. L'attachement à leur territoire d'origine est fort et leur vie quotidienne s'organise le plus souvent dans un périmètre proche et continu.

"Je suis née à Saint-Flour. J'ai grandi à Ussel et je suis toujours à Ussel. J'ai déménagé parce que j'ai déménagé dans Ussel. La maison familiale. Après mes parents ont pris un commerce à Ussel, donc on a déménagé au bar. Et après donc j'ai pris un appartement à Ussel et là j'ai acheté une maison à Luc d'Ussel. C'est juste à côté. » (Femme, 25 ans, Pays de Saint-Flour Haute Auvergne) 
Lorsqu'ils sont amenés à quitter le territoire, c'est souvent de manière temporaire et pour un projet précis : réaliser des études, saisir une opportunité professionnelle « pour mettre l'argent de côté »...

«- Et pourquoi du coup être parti à Lyon?

- Pour la paye tout simplement. " (Homme, 30 ans, Pays de Saint-Flour Haute Auvergne)

La vie en ville n'est pas un objectif en soi, c'est d'ailleurs souvent un type de territoire déprécié. Des caractéristiques, qu'ils jugent négatives, sont mises en avant : la forte densité de population, l'anonymat, l'insécurité... Dans leur récit, ils opposent leurs représentations de la ville - négative - et de la campagne - positive.

"Parce qu'on peut... parce qu'on n'a pas peur non plus de sortir dehors, tsé parce que des fois en ville, les gens sont peut-être des fois plus malveillants mais ici les gens sont accueillants, sont sociables, on dit salut à tout le monde, je trouve que c'est ça qui... Oui, je pense que oui c'est rassurant d'être dans une place où tu sais que les gens sont gentils. » (Femme, 20 ans, Témiscamingue)

Ces jeunes adultes maintiennent des liens denses avec le territoire d'origine lorsqu'ils sont amenés à le quitter. C'est le réseau relationnel de leur ancien lieu de vie qu'ils entretiennent et ils cherchent peu à en recréer un nouveau sur leur territoire d'accueil.

«- On remontait tous les weekends, la semaine on travaillait, le week-end on était là.

- Et vous vous êtes fait des amis là-bas?

- Non du tout.

- Et vos amis venaient vous voir?

- Bah nous on rentrait tous les weekends, donc

le problème était réglé, ils n'avaient pas besoin de venir. » (Homme, 30 ans, Pays de Saint-Flour Haute Auvergne)

L'emploi et la vie de famille (avec leurs enfants, mais aussi avec leurs parents) et l'établissement dans une maison familiale sont des éléments centraux du projet de vie.

\section{Des parcours migratoires comportant des arrivées ou des retours dans les territoires ruraux}

Dans le second type de parcours, le rapport à la ville et à la campagne évolue dans le temps. Les jeunes adultes qui composent cette catégorie sont soit des migrants de retour, soit des nouveaux arrivants dans nos territoires d'étude. Ils associent des « milieux de vie » bien précis aux différentes étapes de leur parcours, de leur vie. À la fin des leurs études secondaires, ces jeunes se montrent avides de quitter le territoire, non seulement pour poursuivre leurs études ou occuper un emploi, mais aussi parce qu'ils souhaitent découvrir de nouveaux horizons et plus particulièrement la ville.

"Oh bah moi j'étais tannée de voir le même monde [...]. Et c'est pour ça que j'ai pas choisi d'aller à Rouyn [...]. J'aurais pu faire mon programme au Cegep à Rouyn aussi sans problème [...]. Moi j'étais contente, à l'âge que j'avais avec les connaissances que j'avais, de le découvrir à cet âge-là. Puis y'a beaucoup de gens qui disent le Témis quand t'es jeune c'est plate tout ça. Moi je trouve ça sain de partir pour mieux revenir. Quand j'avais 17 ans et que je suis partie, j'avais le goût de m'en aller pour voir autre chose. »(Femme, 30 ans, Témiscamingue)

Le départ à cette étape de leur vie est considéré comme normal.

"Bah déjà après ici, sorti de Murat, y'a pas de lycée. À part le lycée professionnel spécialisé dans le bâtiment. Donc déjà pour le lycée t'es obligé de partir un petit peu. Et ensuite, sorti de Saint-Flour, plus autre chose que le lycée. Donc si tu veux faire des études supérieures, c'est Clermont-Ferrand, Aurillac, mais Aurillac y'a pas grand-chose non plus. Donc tous les jeunes d'ici s'ils veulent faire des études supérieures, quoi qu'il arrive ils bougent. » (Homme, 23 ans, Pays de Saint-Flour Haute Auvergne)

Si dans un premier temps, la plupart se rend dans le centre urbain de proximité - Rouyn-Noranda ou Clermont-Ferrand - d'autres, moins nombreux, effectuent un départ plus lointain. Le départ n'est pas vécu comme une contrainte mais comme une opportunité. Pour autant, il n’implique pas nécessairement une rupture des liens avec le territoire 
d'origine. Ceux qui restent à proximité, sont souvent amenés à rentrer le week-end et pendant les vacances. Ils entretiennent alors souvent deux cercles de relations amicales : sur leur territoire d'origine et sur leur territoire d'accueil.

"Mais au CEGEP c'était vraiment différent, je revenais toutes les fins de semaines. Y'a vraiment un système de covoiturage incroyable entre cégépiens, c'était 5 dollars aller et puis on faisait vraiment super commun, on passait toutes nos fins de semaines ici. » (Femme, 28 ans, Témiscamingue)

Pour ceux, qui habitent à une plus grande distance, les retours sont moins fréquents, mais toutefois réguliers. Ils peuvent s'effectuer sur des courtes durées, de type une à deux semaines, pour rendre visite à la famille, aux amis, ou des durées plus longues, lorsque, par exemple, ils occupent un emploi d'été dans le territoire rural.

"L'été, c'est sur que c'est tout le monde qui sont aux études normalement, qui sont revenus. Tsé, à moins de vraiment pas aimer, y'en a qu'aiment pas, qui n'aiment pas vivre ici pour $X$ raison, eux ils ne reviennent pas. Mais moi je trouvais ça dur les étés de pas revenir, parce que je travaillais là-bas, fait que je n'avais pas le choix. Je travaillais dans un restaurant là-bas à Québec, fait que là... Je trowvais ça dur de venir passer deux semaines, je ne trowvais pas ça assez. » (Femme, 27 ans, Témiscamingue)

Après un nombre variable de déménagements, à des distances plus ou moins lointaines selon les études, les projets professionnels, relationnels... propres à chacun, les jeunes ayant ce type de parcours envisagent un retour vers la campagne. Ce retour correspond généralement à une période charnière de leur vie : la fin des études, d'un contrat de travail, la naissance d'un enfant. Au Témiscamingue, le dispositif Place Aux Jeunes ${ }^{7}$ (PAJ), semble jouer, pour certains, un rôle décisif dans la décision de revenir. Il facilite la reprise de contact avec le territoire et notamment avec des employeurs. De retour

7. Place aux jeunes du Témiscamingue est la déclinaison dans la MRC de l'organisme Place aux Jeunes en Région, organisant principalement des "séjours exploratoires » courts (une fin de semaine) ou longs (trois fins de semaines). Ces séjours permettent à de jeunes migrants diplômés de revenir dans leur région d'origine, de découvrir ses potentiels en termes d'activité, d'emploi et de vie culturelle, ainsi que de créer des relations avec des acteurs locaux, dans l'optique de favoriser le retour de jeunes en région. Présent dans 15 régions (sur 17) au Québec, l'organisme propose aussi, au Témiscamingue, un volet de sensibilisation au territoire destiné aux adolescents. sur le territoire, ces jeunes adultes prennent généralement une part active à la vie locale. Le fait d'habiter sur le territoire correspond à un choix de vie. Des nouveaux arrivants possèdent également ce type de parcours. Même s'ils n'ont pas vécu leur enfance sur le territoire, l'arrivée à la campagne est un choix, qui correspond à une étape de leur vie.

\section{Des parcours migratoires qui combinent des modes d'habiter à la ville et à la campagne}

Les parcours migratoires de cette catégorie sont révélateurs de stratégies résidentielles qui combinent des modes d'habiter à la ville et à la campagne. Ils se différencient de la catégorie précédente, dans la mesure où le fait de vivre dans différents types de lieux se combine dans le temps, au lieu de se succéder.

" J'ai quand même une assez grande opposition entre semaine et week-end même si, par exemple, y'a beaucoup de week-end comme ce week-end-là où j'ai un truc à Clermont samedi soir mais je vais quand même rentrer chez moi [dans le Cantal] vendredi, parce que j'ai du travail à faire vendredi soir, samedi dans la journée. » (Homme, 23 ans, Pays de Saint-Flour Haute Auvergne)

Contrairement à la première catégorie également, on ne cherche pas à vivre tout le temps à la campagne, ni à y revenir rapidement et/ou définitivement. Les jeunes ayant un tel type de parcours sont aisément mobiles sur le plan résidentiel mais cherchent à conserver quelques ancrages fixes. Ils ne se considèrent ni comme des urbains, ni comme des ruraux, car ils attribuent des fonctions différentes à chaque espace qu'ils habitent. Ils possèdent (en tant que propriétaires ou locataires), ou bien fréquentent (en rendant visite à de la famille, des amis, pour le travail) alors plusieurs lieux de résidence. L'attachement à ces différentes résidences, mais surtout aux territoires dans lesquels elles se situent, peut varier en fonction des activités qu'ils y mènent. Les territoires ruraux que nous avons étudiés peuvent, dans ce cas de figure, faire office de port d'attache, soit comme une transition entre plusieurs mobilités résidentielles, soit aux côtés d'un ou plusieurs autres lieux de vie. Un point commun entre les jeunes ayant ce type de profil réside dans leur 
capacité organisationnelle et/ou financière à adopter un tel mode d'habiter. Des déplacements fréquents sont nécessaires pour entretenir ce rapport aux territoires. En eux-mêmes, ces derniers peuvent générer des coûts, même s’ils peuvent être diminués par des pratiques comme le covoiturage notamment. Pour les jeunes adultes possédant deux résidences, il convient de considérer les frais liés au logement.

\section{Des parcours migratoires marqués par une crainte de la stabilité résidentielle}

Dans le dernier type de parcours migratoires que nous avons identifiés, la découverte de nouveaux territoires, la mobilité résidentielle, sont des éléments mobilisés de manière récurrente et structurante dans les récits. Les jeunes adultes ayant un tel type de parcours ont pour point commun une certaine crainte de la stabilité résidentielle. Ils disent ne pas avoir envie " de se poser », trouvent cela « apeurant».

«Moi ce qui me fait peur c'est toutes les contraintes de logement. J'ai l'impression qu'à partir du moment où tu as vraiment ton pied-à-terre et tout ça, t'as beaucoup moins de... j'sais pas. Après je paralyse et j'ai un espèce de traumatisme de la stabilité je pense, de tout ce qui est maison à toi, tout ça. Pour moi, ça engendre beaucoup de responsabilités et de contraintes qui font que t'as peut-être moins de possibilités de bouger partout quoi. » (Femme, 24 ans, Pays de Saint-Flour Haute Auvergne)

Leurs parcours sont souvent constitués de nombreuses étapes, les déménagements sont fréquents et souvent lointains, y compris à l'international. C'est le cas par exemple de cette jeune femme âgée de 24 ans au moment de l'entretien. Après avoir quitté le Cantal pour suivre des études à Clermont-Ferrand puis à Lyon, elle enchaîne des séjours à l'étranger : Mexique, Palestine, Espagne, Mauritanie. Les motivations au départ reposent beaucoup sur des envies dites de «découvertes», de dépaysement, d'apprentissage de langues étrangères, mais aussi pour rencontrer de nouvelles personnes ou rejoindre des amis. Dans ce type de parcours migratoires, les jeunes adultes se distinguent des touristes. Ils ne sont pas là uniquement pour visiter des lieux, mais souhaitent s'y impliquer et pérenniser leurs réseaux relationnels. Les durées de séjours s'étalent en général sur plusieurs mois ou années. Afin que cela soit possible, les jeunes adultes sont souvent mobiles avec un statut qui leur permet de prendre une place dans les territoires qu'ils habitent : étudiants, volontaires, travailleurs... Ils cherchent aussi souvent à s'impliquer dans les réseaux associatifs ou militants. Il y a des moments où ils choisissent leurs destinations en fonction de leurs projets et d'autres où ils configurent leurs projets en fonction de l'endroit qu'ils souhaitent rejoindre.

\section{DISPOSITIFS TERRITORIAUX EN FAVEUR DE L'ACCUEIL, DU MAINTIEN ET DE LA MOBILITÉ DES JEUNES ADULTES DANS LES TERRITOIRES RURAUX}

Les jeunes adultes possèdent, comme nous venons de le voir, un rapport complexe à la mobilité (avérée ou potentielle) et aux territoires. Les dispositifs mis en œuvre dans les territoires ruraux prennent-ils en compte cette complexité?

\section{Les territoires ruraux face aux mobilités des jeunes adultes}

Dans le Pays de Saint-Flour Haute Auvergne et au Témiscamingue, la majorité des actions, à destination des mobilités ou de l'accueil des jeunes adultes, vise d'une part à favoriser leurs retours ou leurs arrivées et d'autre part à pérenniser leurs installations. De nombreux dispositifs, dont certains sont présentés dans les paragraphes suivants, sont mis en œuvre et mobilisent une diversité d'acteurs à l'échelle du territoire, mais aussi des régions dans lesquelles ils s'inscrivent. Si globalement elles se chargent de promouvoir l'ensemble de la région, à l'extérieur, le Pays ou la MRC mettent en œuvre des actions plus ciblées à destination de personnes souhaitant s'installer.

\section{Favoriser les arrivées et les retours, pérenniser les installations}

Dans le Pays de Saint-Flour Haute Auvergne, les potentiels nouveaux arrivants ciblés par les dispositifs institutionnels sont surtout « les porteurs de projets », des personnes souhaitant créer leur propre activité ou emploi ou reprendre une entreprise exis- 
tante. Aussi, les jeunes adultes ne constituent-ils pas une « cible» identifiée des dispositifs en œuvre. Les nouveaux arrivants, jeunes ou non, peuvent bénéficier d'accompagnement au montage de leur projet professionnel - le plus souvent en termes de création d'activité - ainsi que d'une présentation du territoire, et cela même en amont de leur installation. Des sessions d'accueil d'actifs sont ainsi organisées deux fois par an à l'échelle des communautés de communes du département du Cantal. La région Auvergne a également mis en place un dispositif de résidence d'entreprise permettant de démarrer ou de tester des projets d'activités. En parallèle de l'accompagnement ou de l'accueil des personnes souhaitant s'installer, le Pays de Saint-Flour Haute Auvergne travaille également sur les conditions d'accueil du territoire et notamment sur la dimension habitat logement et services à la population -, qu'ils cherchent à améliorer, tant dans une optique d'attractivité du territoire que de maintien de la population. Afin de favoriser le maintien des habitants dans le territoire, la chargée de mission « accueil » du Pays souligne le rôle clé des élus locaux pour s'assurer que les nouveaux arrivants sont bien installés, mais aussi celui de l'accueil réservé par les autres habitants et de la capacité même de la personne à faire sa place dans le territoire.

En Abitibi-Témiscamingue, il y a une forte préoccupation pour recruter une main-d'œuvre qualifiée ou non afin de pourvoir les emplois vacants. L'accent n'est pas mis de façon aussi prégnante sur les porteurs de projets, de créateurs d'activité ou d'entreprises, même si ces derniers peuvent également être accueillis de manière spécifique par les territoires. La région de l'Abitibi-Témiscamingue ainsi que l'organisme PAJ du Témiscamingue cherchent tout particulièrement à accueillir des jeunes adultes. Pour ce faire, deux dispositifs phares sont mis en œuvre. Il s'agit tout d'abord d'inciter des étudiants finissant leurs études à revenir ou à découvrir les offres d'emploi du Témiscamingue. Les agents de migration ${ }^{8}$ de la région participent à une tournée de promotion du territoire dans les centres urbains du Québec (Montréal, Sherbrooke, Québec, Trois Rivières). Cette tournée ( $1^{\text {er }}$ dispositif) s'appelle « l'Abitibi-Témiscamingue débarque! »PAJ du Témiscamingue organise par ailleurs, deux fois par

8. Les personnes travaillant pour l'organisme Place aux Jeunes en Région sont appelées « agents de migration ». an, des séjours exploratoires (2e dispositif), qui permettent à des étudiants de venir découvrir le territoire (par des visites touristiques, de lieux culturels, d'entreprises...), de rencontrer des employeurs et de prendre ou reprendre contact avec des habitants (témoignages de gens s'étant installés ou étant revenus, soirées festives avec des jeunes adultes habitant le Témiscamingue...). Au Témiscamingue, on observe aussi une forte préoccupation des acteurs du territoire (élus et acteurs intermédiaires) à retenir les habitants, comme en témoigne la planification stratégique du Témiscamingue, faisant de l'attractivité, mais aussi du maintien ${ }^{9}$ de la population, un axe de développement. Sans prétendre à l'exhaustivité, nous relatons trois initiatives veillant à ce que l'intégration des nouveaux arrivants soit facilitée. La première consiste en la création d'un comité d'accueil des nouveaux arrivants, composé principalement des «employeurs ou des gens qui font une différence sur le territoire » (Acteur intermédiaire, Témiscamingue): la MRC du Témiscamingue, la commission scolaire, le Centre local d'emploi, le Centre de santé et de services sociaux, la corporation Augustin Chénier ${ }^{10}$, le Carrefour Jeunesse Emploi. Tous les nouveaux arrivants ne transitent pas par des dispositifs institutionnels permettant aux acteurs organisés pour l'accueil de prendre connaissance de leur venue. Les membres du comité d'accueil veillent donc à ce que les nouveaux arrivants soient bien accueillis dans leur milieu de travail, soient mis au courant des opportunités de découvertes et d'expériences au Témiscamingue de manière plus générale. Ils informent l'agente de participation citoyenne ${ }^{11}$, aussi chargée de l'accueil des nouveaux arrivants, de l'arrivée des nouveaux venus. Celle-ci envoie ensuite un courriel pour proposer aux personnes de la rencontrer, afin de discuter de leurs envies et besoins sur le territoire. La seconde initiative, ponctuelle, est inspirée d'habitudes prises par certaines municipalités d'accueillir de façon directe les nouveaux arrivants, en leur offrant un cadeau de bienvenue, en les présentant à la fête du village... Afin de

9. Au Québec, le terme employé pour désigner le maintien est celui de « rétention".

10. La corporation Augustin Chénier, regroupe au Témiscamingue un théâtre et une salle d'exposition.

11. Chaque région du Québec est dotée d’un forum jeunesse au sein duquel travaille un agent de participation citoyenne. Au vu de l'étendue de la Région Abitibi-Témiscamingue, il a été décidé, en lien avec les Carrefours Jeunesse Emploi de créer un poste d'agent de participation citoyenne dans chacune des MRC de la Région. 
répandre cette pratique à l'ensemble du territoire, le comité d'accueil des nouveaux arrivants travaille à la création d'une pochette d'accueil qui sera remise par toutes les municipalités à chacun des nouveaux arrivants. Elle contiendra des informations sur la municipalité choisie, ainsi qu'un DVD retraçant le parcours croisé d'un nouvel arrivant et d'un témiscamien de longue date. Enfin, si les initiatives du comité d'accueil sont nées pour dynamiser le milieu et le sensibiliser à l'accueil des nouveaux arrivants, il est rapidement apparu que les activités proposées par ce dernier n'étaient pas suffisamment fréquentes pour permettre aux nouveaux arrivants de tisser des liens sociaux entre eux. C'est pourquoi, les nouveaux arrivants, eux-mêmes, se sont constitués en comité afin de faciliter l'intégration au milieu.

\section{Laisser partir, mais permettre de revenir}

Concernant le départ des jeunes depuis les territoires, le discours des élus locaux ou des acteurs intermédiaires est ambivalent. Les départs sont considérés comme un problème. On cherche à maintenir des jeunes sur le territoire, parfois pour contrer des menaces de fermeture d'établissements scolaires faute d'effectifs. Même s'ils considèrent les départs comme une contrainte pour le développement, les élus locaux ou les acteurs intermédiaires que nous avons rencontrés, les considèrent aussi comme une opportunité pour les individus.

"Je pense que le départ est une étape importante, parce que la réalité de notre territoire fait qu'on n'a pas accès à la formation à tous les niveaux et tous les types de formation chez nous. Donc forcément un jeune qui veut une formation de façon plus précise doit quitter la région de toute façon. Et puis en même temps, le fait de quitter la région, souvent aussi ça amène un regard nouveau, ça amène une dynamique nouvelle. » (Acteur intermédiaire, Témiscamingue)

Bien que les départs du territoire apparaissent comme une difficulté, le maintien n'apparaît pas comme la solution la plus pertinente. Plutôt que de retenir, il convient de mettre en ouvre les conditions favorables aux retours.

"Le risque c'est que les jeunes partent, fassent leur vie ailleurs et ne reviennent pas. Je dirais, ce n'est pas grave en soi, l'important c'est que le jeune il puisse vivre sa vie. Par contre en termes de territoire, c'est compliqué parce que l'ouverture à de jeunes populations qui viennent de l'extérieur n'est pas toujours si facile que ça. » (Élu, Pays de SaintFlour Haute Auvergne)

Le départ du territoire est en fait considéré comme une sorte d'étape logique des parcours individuels des jeunes adultes, face auxquels les acteurs publics des territoires ruraux n'ont pas ou peu de rôle à jouer.

\section{Accompagner les départs}

Dans le Pays de Saint-Flour Haute Auvergne, le territoire n'intervient pas dans l'accompagnement du départ des jeunes adultes. Les choix d'orientation se font au niveau individuel, dans le cadre familial ou par l'intermédiaire des établissements scolaires. Si les jeunes adultes font des choix qui les amènent à s'installer dans une autre région, les acteurs du développement ne gardent pas le contact. Au Témiscamingue, en revanche, l'organisme PAJ a mis en place un dispositif à destination des adolescents sur le point de quitter le territoire à la fin de leurs études secondaires. Organisé sur le temps scolaire et composé de deux volets, il consiste d'une part à apporter aux élèves des éléments de connaissance sur leur région, des témoignages de jeunes adultes revenus s'installer au Témiscamingue de manière à en faire des « ambassadeurs » lorsqu'ils habiteront dans un autre territoire. D'autre part, l'agent de migration territorial recueille les contacts des élèves, de manière à les tenir informés à distance, via des envois de courriels, des évolutions du territoire, en termes de cadre de vie, d'opportunités d'emploi ou d'événements culturels, lorsqu'ils sont partis.

"Ben en fait, Place aux Jeunes Ados prépare un peu [le départ du territoire], enfin rappelle aux jeunes dans le fond qu'ils vont partir. Ça leur rappelle qu'il faut qu'ils fassent des choix et que nécessairement ils ne seront pas au Témiscamingue. Mais nous, c'est plus dans la sensibilisation au niveau $d u$ sentiment d'appartenance et tout, c'est qu'ils soient aussi de bons ambassadeurs de leur région! Qu'ils parlent de leur région positivement. » (Acteur intermédiaire, Témiscamingue) 


\section{Des initiatives discrètes pour favoriser les apprentissages par la mobilité}

Lors des entretiens, certains acteurs intermédiaires et élus locaux se sont montrés sensibles aux difficultés rencontrées par certains jeunes adultes pour quitter le territoire. Selon eux, ces jeunes adultes manquent de repères, de compétences ou d'habitudes. Ces difficultés sont considérées comme un problème, dans la mesure où elles ne permettent pas aux individus de développer leurs projets ou de s'impliquer comme ils le souhaiteraient sur le territoire, les conduisant à y rester de manière subie et non choisie. Les élus ou acteurs intermédiaires nous parlent tout particulièrement de jeunes adultes, principalement dans les filières courtes et professionnalisantes, ou se trouvant éloignés du marché de l'emploi. Dans le Pays de Saint-Flour Haute Auvergne et au Témiscamingue, deux expériences visant à dépasser ces situations, jugées difficiles, par un projet de mobilité à l'international nous ont été relatées ${ }^{12}$.

La première expérience, au Témiscamingue, consiste à accompagner un groupe de jeunes âgés de 18 à 35 ans sur la préparation et la réalisation d'un voyage au Honduras. Portée par le Carrefour Jeunesse Emploi, cette initiative vise à leur permettre de développer des compétences dans différents domaines pour favoriser le retour à l'emploi ou aux études : découverte d'une langue étrangère, montage de projet, travail en équipe..., à favoriser la reprise de confiance en soi et à susciter des envies nouvelles.

"Donc de le faire [la préparation + le voyage] à travers ça, ça permet un nouvel apprentissage, de nouvelles énergies peut-être puis dans le fond, travailler des éléments nouveaux, soit de l'emploi ou d'un retour aux études, mais la mise en mouvement [...]. Fait qu'à travers ce projet-là, les jeunes apprennent les éléments de la culture entrepreneuriale, sans trop s'en rendre compte, parce qu'on va participer à des levées de fond, parce qu'on va monter un projet, parce qu'on va travailler en équipe, parce qu'on va découvrir la gestion du budget, tsé y'a plusieurs habiletés qui vont se découvrir sans qu'on ait à mettre un titre [...]. Là-bas à l'étranger, ben c'est une énorme prise de confiance de soi [...]

12. Nous ne sommes pas exhaustifs sur la diversité des initiatives de ce genre ayant cours dans nos territoires d'étude. parce que pour plusieurs de ces jeunes-là, ils n'ont jamais quitté nécessairement, soit la région, soit le Québec et puis encore moins été à l'international, fait que c'est souvent des gros défis qui font que au retour les jeunes sont fiers d'avoir réalisé. " (Acteur intermédiaire, Témiscamingue)

Dans le Pays de Saint-Flour Haute Auvergne, un groupe de 12 jeunes âgés de 18 à 23 ans, élèves du lycée professionnels du bois de Murat, participent à un projet européen de festival de cinéma itinérant entre cinq pays et établissements scolaires (France, Belgique, Allemagne, République Tchèque et Turquie). Selon l'enseignante à l'initiative du projet, la plupart des jeunes impliqués étaient, au départ, réticents à l'idée de quitter le territoire, par peur de ne pas en avoir les capacités.

«Moi je ne viens pas de lycée général. Je travaille dans un lycée professionnel. C'est deux publics complètement différents. En lycée professionnel, pour les élèves qui viennent du territoire, parce que je n'ai pas que des élèves qui viennent du territoire, mais pour les élèves, on va dire, qui viennent $d u$ Cantal, s'expatrier c'est souvent douloureux. En tout cas c'est inquiétant. Parce qu'ils ont l'impression de ne pas maîtriser les outils et donc il y en a qui se brident et qui vont modifier leur carrière ou leur projet professionnel pour ne pas avoir à quitter le territoire, parce que pour eux ce n'est pas possible quoi. » (Enseignante, Pays de Saint-Flour Haute Auvergne)

Comme dans le projet précédent, l'objectif de cette expérience est de donner aux jeunes une capacité d'agir, pour, par la suite, prendre place dans les dynamiques sociales, culturelles ou économiques du territoire avec un regard neuf, ou tout au moins de ne pas y rester de manière subie.

\section{LA MOBILITÉ DES JEUNES ADULTES : UNE RESSOURCE POUR LES TERRI- TOIRES RURAUX?}

Les résultats présentés ci-avant donnent à voir que les mobilités des jeunes adultes sont multiples et se combinent de façon différenciée avec l'ancrage territorial et la participation aux dynamiques sociales, économiques, environnementales ou culturelles des territoires qu'ils habitent. Parallèlement à ces mobi- 
lités résidentielles, qui relèvent de l'individu et de son environnement, les politiques mises en place par les territoires favorisent principalement l'accueil ou le retour des jeunes. Dans cette dernière partie, nous discutons ces résultats en nous demandant s'il est possible de changer le regard réciproque et les pratiques des différents acteurs pour une plus grande mise en dynamique.

\section{Rôle des territoires dans l'augmentation du capital motilité des jeunes adultes}

En mettant la focale sur le parcours migratoire des jeunes, c'est-à-dire en nous intéressant aux différents lieux habités au cours de leur vie et aux éléments ayant motivé ou non les changements, nous avons montré que la mobilité résidentielle des jeunes adultes, contrairement à ce qu'ont longtemps laissé entendre les discours sur l'exode rural, n'est pas un phénomène linéaire qui conduit nécessairement, et de manière irréversible, les jeunes de la campagne à la ville (LeBlanc, 2005). Les jeunes adultes mobilisent les caractéristiques des territoires - urbains comme ruraux - pour justifier ou organiser leur vie quotidienne, leurs choix résidentiels, actuels ou futurs (Gambino, 2008). Ils font aussi partie des migrants qui s'installent dans les territoires ruraux (Cognard, 2001). Même si les jeunes adultes sont plus nombreux que les autres tranches d'âge à déclarer vouloir quitter la campagne pour la ville (PerrierCornet, 2002), les circulations sont complexes et multiples, les retours et arrivées sont envisageables (Gaultier et al., 2006). Partir d'un territoire rural car on emménage ailleurs n’implique pas nécessairement une rupture des liens et peut même renouveler l'ancrage et la manière de s'y impliquer.

Pourtant, « le discours contemporain du rural [est] positif et séduisant, [mais] ne doit pas masquer les inégalités liées à la mobilité dont il est aussi un révélateur. » (Perrier Cornet, 2002). Tous les jeunes adultes ne sont pas égaux dans leur capacité à être mobiles et a fortiori à s'y appuyer pour développer des projets pour eux-mêmes ou pour le territoire. Certaines personnes subissent la mobilité ou la sédentarité. Afin de mieux comprendre les relations que les jeunes adultes entretiennent aux mobilités, mais également pour éviter des irréversibilités socio-spatiales, la notion de motilité, développée par Kaufman et Jemelin (2004) nous semble pertinente à la fois pour l'analyse du phénomène et la mise en œuvre de dispositifs territoriaux répondant aux problèmes constatés.

Pour ces auteurs, la notion de motilité recouvre non seulement les déplacements avérés, mais aussi «potentiels». La notion se compose de trois dimensions (Kaufman et Jemelin, 2004) : l'accessibilité, la compétence et l'appropriation. «L'accessibilité renvoie à la notion de service, il s'agit de l'ensemble des conditions économiques et spatio-temporelles auxquelles une offre de déplacement et de communication peut être utilisée » (existence d'une ligne de train, possession d'une voiture, horaire, tarif...). « Les compétences relèvent de la socialisation et reposent sur deux aspects centraux dans la dimension des compétences : les savoir-faire acquis permettant de se déplacer et les capacités organisationnelles, comme la manière d'agencer ses activités dans le temps et l'espace, ou la manière de les planifier (programmation, réactivité...). » L'appropriation, quant à elle, est « le sens donné par les acteurs aux possibilités de mobilité auxquelles ils ont accès. C'est la manière dont les individus évaluent leurs possibilités d'être mobiles. L'appropriation relève donc de stratégies, valeurs, perceptions et habitudes. Elle se construit notamment par l'intériorisation de normes et de valeurs ». Pour Kaufman et Widmer (2005), la motilité est un capital qui s'acquiert pour une bonne partie dans le cadre de la famille, et qui, comme les autres capitaux, peut-être source d'inégalités entre les individus.

Construire des dispositifs permettant aux jeunes adultes d'accéder aux trois dimensions de la motilité est selon nous un moyen de faire en sorte qu'ils ne se sentent ni «piégés » dans les territoires ruraux (Gambino, 2008) ni par des mobilités résidentielles subies. La dimension de l'accessibilité est souvent le point central des dispositifs en œuvre sur les territoires : transport à la demande, tarifs préférentiels pour les déplacements par train en région, en fournissant des aides au permis de conduire... Les dimensions « compétences » et « appropriation » ne sont que rarement prises en compte. Il nous semble alors que c'est l'expérience du départ, du déplacement, qui permet de les développer. Certains jeunes adultes ont été amenés, dans leur enfance ou leur adolescence par exemple, à être mobiles pour diverses raisons (déménagement, vacances, visites à la famille...). Lors de ces déplacements, 
ils ont pu développer des compétences nécessaires à devenir mobiles, de façon autonome : prendre le train, le métro, combiner les modes de transports, organiser un séjour... L'expérience du départ contribue aussi probablement, à démystifier l'ailleurs, le " trop loin ». Ce sont là les effets recherchés par les acteurs intermédiaires à l'origine des initiatives discrètes présentées ci-avant, qui gagneraient probablement à être plus soutenues dans les dispositifs territoriaux afin de permettre à l'ensemble des jeunes adultes de développer leur capital motilité.

\section{Contribution de la mobilité des jeunes au capital spatial des territoires}

Au travers de leurs parcours migratoires, les jeunes adultes tissent des liens entre les différents territoires qu'ils habitent. Par le biais de leurs mobilités, notamment résidentielles, les jeunes adultes contribuent à forger l'image du territoire à l'extérieur. Ils en parlent et le présentent à d'autres qui ne le connaissaient peut-être pas auparavant. Pour Moquay (1997) comme pour les tenants du dispositif PAJ Ados, les jeunes adultes qui quittent le territoire deviennent les ambassadeurs de leur région car ils contribuent à diffuser une image de cette dernière. En partant, c'est également leurs propres représentations du territoire qui évoluent. La mise à distance du territoire est parfois même à l'origine d'une prise de conscience d'un attachement au territoire d'origine (Jamet, 2011 ; Guérin-Pace, 2006; Garneau, 2003).

Ce constat nous semble aller dans le sens de ce que Lévy (2013) décrit comme le «capital spatial des territoires ». Pour lui, ce dernier comporte non seulement des éléments qu'il nomme «objectifs", comme « leur localisation, leurs liens avec d'autres lieux, les réalités qu'ils contiennent ", mais aussi des éléments plus «subjectifs comme leur image sur place ou à l'extérieur, l'identité spatiale de ses habitants ou encore la capacité de mobilisation de la société sur un projet » (Lévy, 2013).

Parallèlement aux actions conduites pour favoriser les arrivées et les retours, les territoires ruraux semblent avoir intérêt à questionner et travailler la question des départs, de manière à ce qu'ils ne se posent plus comme une contrainte mais comme une ressource pour le développement. Dès lors, il s'agit d'envisager que les temps des départs et des arrivées ne soient plus opposés mais combinés au sein de politiques globales de mobilité. Plutôt que de les configurer en interne des périmètres administratifs, n'est-il pas nécessaire de concevoir des politiques de mobilité, considérant, dès le départ, des liens entre territoires?

\section{Conclusion}

Au travers des récits de leurs parcours migratoires, les jeunes adultes donnent à voir, dans nos deux terrains d'étude essentiellement ruraux et éloignés des grands centres urbains, des rapports complexes aux territoires et aux mobilités. Pour la plupart des jeunes, arrivées et départs sont des éléments qui se combinent, plus qu'ils ne s'opposent. Cependant, tous les jeunes adultes ne sont pas égaux face aux mobilités. Si certains sont en capacité de mettre leurs mobilités au service de leurs projets personnels, professionnels, familiaux... ou de projets pour le territoire, d'autres - souvent plus fragiles - la subissent et se sentent alors soit contraints de partir, soit contraints de rester. S'il ne s'agit pas d'inciter les jeunes adultes à quitter les territoires ruraux, ils convient de permettre à chacun d'avoir le potentiel de le faire, de manière à ce que le fait d'habiter le territoire soit non plus un état subi mais choisi, que l'installation se fasse sans ou suite à une mobilité résidentielle préalable. Pour cela, les dispositifs territoriaux doivent combiner les trois dimensions de la motilité (Kaufmann, 2005) que sont l'accessibilité, les compétences et l'appropriation.

Pour les élus et acteurs intermédiaires, les mobilités des jeunes adultes - et notamment lorsqu'ils quittent le territoire - sont sources d'inquiétude car la croissance démographique est considérée comme un gage du développement territorial. De ce fait, ils concentrent l'essentiel de leurs actions à favoriser les retours ou les arrivées dans leurs territoires. Pourtant, le départ des jeunes adultes peut s'avérer comme une ressource, non seulement pour les jeunes eux-mêmes mais aussi pour les territoires. La mise à distance du territoire permet aux jeunes de se former, d'acquérir des connaissances et compétences, de développer des réseaux sociaux qu'ils n'auraient pu avoir en restant sur place. Le départ est parfois à l'origine de la prise de conscience d'un sentiment d'attachement, et peut, de temps à autre, déboucher sur un retour ou permettre la conserva- 
tion d'un ancrage solide, d'une fréquentation régulière voire d'une implication dans la vie locale. Le capital spatial des territoires (Lévy, 2013), renforcé par les mobilités des jeunes adultes, lui donne une visibilité depuis les autres régions.

\section{Bibliographie}

Barthe, L., Milian, J., 2011 . Les espaces de la faible densité - état des lieux et problématiques, Territoires 2040, n 3 , p. 141-148.

Bertaux D., 2010. L'enquête et ses méthodes: le récit de vie, Paris, Armand-Colin, coll. « 128 », 128 p.

Olivier David, Le temps libre des jeunes ruraux : des pratiques contraintes par l'offre de services et d'activités de loisirs, Territoire en mouvement Revue de géographie et aménagement [En ligne], 22|2014, mis en ligne le 15 juin 2014, consulté le 25 août 2014. URL : [http://tem.revues.org/2423].

Cognard F., 2001. Reprise démographique et nouvelles populations dans les moyennes montagnes françaises, Espaces, Populations, Sociétés, vol. 19, n 1-2, p. 53-68.

Cognard F., 2010. «Migrations d'agrément » et nouveaux habitants dans les moyennes montagnes françaises: de la recomposition sociale au développement territorial. L'exemple du Diois, du Morvan et du Séronais, Thèse de doctorat en géographie, Université Blaise-Pascal, Clermont-Ferrand, 528 p.

Gambino M., 2008. Vivre dans les espaces ruraux de faible densité de population : pratiques et représentations des jeunes dans le Périgord vert (France) et le rural Galway (Irlande), Thèse de doctorat en géographie-aménagement, Université de Toulouse, $376 \mathrm{p}$.

Gambino M., 2010. Les mobilités géographiques des jeunes dans les espaces ruraux de faible densité. Centre d'études et de prospectives, $\mathrm{n}^{\circ} 22,8 \mathrm{p}$.

Garneau S., 2003. La mobilité géographique des jeunes au Québec : la place du territoire, Recherches sociographiques, vol. 44, n 1 , p. 93-112.

Gaultier M., LeBlanc P., Côté S., Deschenaux F., Girard C., Laflamme C., Magnan M.-O., Molgat M., 2006. La migration des jeunes au Québec. Rapport national d'un sondage 2004-2005 auprès des 20-34 ans du Québec, 185 p.

Guerin-Pace F., 2006. Lieux habités, lieux investis : le lien au territoire, une composante identitaire?, INSEE - Économie et Statistiques, vol. 393-394, p. 101-114.

Guerin M., 2001. Propos introductifs, in Guérin M., Vollet D., Territoires ruraux et nouveaux venus, Aubière, ENITA, coll. «Acte, p. 15-17.

Guimond L., 2012. Lorsque les nouvelles populations rurales rencontrent les plus anciennes: l'expérience géographique au cœur de la nouvelle ruralité an Québec, Thèse de doctorat en géographie, Université d'Ottawa, 210 p.

Insee, 2013a. Population légale 2010, Cantal. Repères n 51, 2 p.: [http://www.insee. fr/fr/themes/document.asp?reg_ $i d=10$ Eref_id=19403], consulté le 31 août 2014.
InSEE, 2013b. Le Pays de Saint-Flour Haute Auvergne face à de nouveaux défis d'aménagement territorial. INSEE Auvergne, La lettre $\mathrm{n}^{\circ}$ 91, $4 \mathrm{p}$.

InSEE, 2009. RP2009 exploitation principale.

Institut de la Statistique du Québec, 2014. 08 - L'AbitibiTémiscamingue ainsi que ses municipalités régionales de comté (MRC) : [http://www.stat.gouv.qc.ca/statistiques/profils/region_08/region_08_00.htm], consulté le 31 août 2014.

Jameт E., 2011. La migration des jeunes depuis les villes intermédiaires, une ressource pour les régions rurales? Réflexions à partir d'une étude de cas de deux villes intermédiaires au Québec et en Colombie Britannique, in Giroud M. et al., Les mobilités spatiales dans les villes intermédiaires, territoires, pratiques, régulations. Actes du colloque de Clermont-Ferrand, 25 et 26 novembre 2010., Presses Universitaires Blaise-Pascal, coll. « Ceramac » p. 273-290.

JEAN B., 2006. Présentation : le développement territorial : un nouveau regard sur les régions du Québec, Recherches sociographiques, vol. 47, p. 465-474.

Kaufmann J.-C., 2007. L'enquête et ses méthodes: L'entretien compréhensif, Paris, Armand Collin, coll. «128 », 128 p.

Kaufmann V., Jemelin C., 2004. La motilité, une forme de capital permettant d'éviter les irréversibilités socio-spatiales?, Colloque de géographie sociale «Espaces et Sociétés aujourd'hui », Rennes.

Kaufmann V., 2005. Mobilités et réversibilités : vers des sociétés plus fluides?, Cahiers internationaux de sociologie, vol. 118, p. 119-135.

Kaufmann V., Wiedmer E.-D., 2005. « L'acquisition de la motilité au sein des familles. État de la question et hypothèses de recherche », Espaces et Sociétés, vol. 120-121, p. 199-217.

LeBlanc P., 2005. Les mythes de l'exode des jeunes. Les deux tiers des jeunes vivent dans leur région d'origine in L'annuaire du Québec 2006. Montréal, Fides, p. 164-173.

LeVY J., 2013. France, une société urbaine, in LAurent E. (dir.), Vers l'égalité des territoires. Dynamiques, Mesures, Politique. Rapport remis au ministère de l'égalité des territoires et du logement.

Moquay P. 1997. Le sentiment d'appartenance territoriale, in Gauthier M et al., Pourquoi partir? La migration des jeunes d'hier et d'aujourd'hui, IQRC (PUL), p. 243-256.

PAillé P., 20Io. Une «enquête de théorisation ancrée »: les racines et les innovations de l'approche méthodologique de Glaser et Strauss, in Glaser B.-G., Strauss A.-A., La découverte de la théorie ancrée. Stratégies pour la recherche qualitative, traduit de l'anglais (américain) par Marc-Henry Soulet et Kerralie Euvray Paris, Armand Colin, coll. « Individu et Société », 409 p.

Perrier Cornet P., 2002. Les Français et l'espace rural. Perceptions communes et pratiques différenciées, Économie et Humanisme, $\mathrm{n}^{\circ} 362$, p. 26-30.

Pistre P., 2011 . Migrations résidentielles et renouveaux démographiques des campagnes françaises métropolitaines, Espaces, Populations, Sociétés, n 3, p. 539-555.

Pistre P., 2012. Renouveau des campagnes françaises. Évolutions démographiques, dynamiques spatiales et recompositions 
sociales. Thèse de doctorat en géographie, Université Paris Diderot, Paris 7, $407 \mathrm{p}$

Robineau O., 2013. Vivre de l'agriculture dans la ville africaine. Une géographie des arrangements entre acteurs à Bobo-Dioulasso, Burkina Faso. Thèse de doctorat en Géographie et Aménagement de l'Espace, Université Montpellier PaulValéry, Montpellier 3, 365 p.

Roussel V., Mamdy J.-F., 2001. Nouveaux arrivants et territoires ruraux fragiles : quelques exemples dans le Massif central, Espaces, Populations, Sociétés, vol. 19, n 1-2, p. 109-122.
Soulet M.-H., 2010. Pourquoi traduire "The discovery of Grounded Theory? ", in Glaser B.-G., Strauss A.-A., La découverte de la théorie ancrée. Stratégies pour la recherche qualitative, traduit de l'anglais (américain) par Marc-Henry Soulet et Kerralie CEuvray Paris, Armand Colin, coll. « Individu et Société », 409 p.

Statistique Canada. 2002. Série de document de travail sur l'agriculture et le milieu rural N 61, n² 21-601-M au catalogue.

Vigour C., 2005. La comparaison dans les sciences sociales. Paris, La Découverte, 336 p. 